\title{
Transparency Theory: Empirical Issues and Psycholinguistic Routes.*
}

\author{
Emmanuel Chemla
}

Laboratoire de Sciences Cognitives et Psycholinguistique, EHESS / CNRS / DEC-ENS, Paris, France.

Draft: July, 7th.

To appear in Theoretical Linguistics as a commentary on Schlenker (2008a).

\section{Achieving explanatory force}

The transparency theory of presupposition projection advocated by Schlenker (2008a) relies on two fairly independent assumptions. First, it describes the interaction between two general principles given in (1) and (2). These two new sub-maxims of manner together govern the use of explicit conjunctions and complex meanings (presuppositional items) to package information.

(1) Be Articulate:

In any syntactic environment, express the meaning of an expression $\underline{d} d^{\prime}$ as ( $d$ and $\left.\underline{d} d^{\prime}\right){ }^{1}$

(2) Be Brief (in brief):

A predicative or propositional occurrence of $(d$ and $x)$ is infelicitous in a position where $d$ and is useless (i.e. in a position where any two expressions of the form $(d$ and $\beta$ ) and $\beta$ lead to contextually equivalent sentences).

These maxims lead to the following prediction: ${ }^{2}$

(3) General prediction of the transparency theory:

A sentence of the form $\varphi\left(\underline{d} d^{\prime}\right)$ presupposes that $\forall \beta \in \mathcal{L}, \varphi(d \wedge \beta) \Leftrightarrow \varphi(\beta)$.

Interestingly, one can show that in the propositional case, this prediction is equivalent to $\varphi(d) \Leftrightarrow \varphi(\top)$, i.e. we only need to consider one possible sentence completion $\beta$ : the tautology. Importantly however, this is only a (very efficient) technical shortcut and lengthy tautologous pieces are not necessary (see Chemla, 2006). This first part of the theory accounts for the core projection facts: projection under negation, conditionals, quantified sentences etc.

Second, there is a processing module which governs the stage at which these Gricean mechanisms apply:

(4) Be Brief may take into account the whole sentence or it may abstract away from the end of the sentence and only take into account what precedes the (structural) position at which the presupposition trigger occurs.

*Despite thanks I owe to Márta Abrusán, Bart Geurts and Benjamin Spector for discussions about this manuscript, errors herein remain my own responsibility.

${ }^{1}$ Notations: The underlined parts stand for presuppositional pieces of meaning.

${ }^{2}$ Notations: $\varphi(\ldots)$ represents a generic environment where presuppositional material like $\underline{d} d^{\prime}$ can be embedded. $\mathcal{L}$ represents the set of expressions of the appropriate type in the language. 
This second insightful hypothesis introduces some optionality in the system, which thus can capture usual presuppositional linear asymmetries. In short, the effects of presupposition triggers can be neutralized when they appear after the justification of their presupposition. For instance, if a presupposition trigger appears in the second half of a conjunctive sentence, and if the first conjunct entails the resulting presupposition, no presupposition is projected for the overall sentence, as in the following caricatural example:

(5) It is raining and Mary knows it/that it is raining.

Empirically speaking, the transparency theory is equivalent to the earliest and most robust versions of dynamic semantics such as Heim (1983) for a very wide variety of cases (full proof in Schlenker, 2007). This empirical success is achieved without any assumption about the environments in which presupposition triggers may appear. The outcome only depends on the standard bivalent meanings of the various parts of the sentences involved: no speculation about, e.g., negations or conjunctions is needed (this criticism of dynamic approaches was already discussed in, e.g., Soames, 1989).

Let me illustrate this point with a slightly less usual example. The exact semantics of conditional sentences is a matter of debate, and it is standard methodology to give them the semantics of material implication: the meaning of If $a, b$ is close to the meaning of ( $\neg a$ or $b$ ). From this approximation, the dynamic meaning of a conditional could be derived from the dynamic meanings of negation and disjunction.

This is bad methodology for at least two reasons. First, there is more than one way to emulate the truth-conditions of material implication with negations and disjunctions - e.g., $(\neg a$ or $b),(b$ or $\neg a)$. The problem is that each of these translations may lead to a different dynamic meaning for the overall expression so that the link between the conditional and the relevant translation needs to be motivated independently. ${ }^{3}$ Second, conditional sentences are not material implications to begin with. The closeness in truth-conditions does not imply that there is any level of representation at which the two expressions are built from similar subcomponents (e.g., negation and disjunction). The situation is rather different with a semantically predictive algorithm like, e.g., the transparency theory. The input of such systems is the bivalent meaning of an expression, ${ }^{4}$ the output is its presuppositional behavior. If we want an approximation of the output, it is fair to use an approximation of the input, i.e. an approximation of the truth-conditions, because no intermediate level of representation is needed before we can apply the algorithm. More importantly, any refined semantics for the conditionals could just as well feed the algorithm and lead to testable predictions. ${ }^{5}$

So, the transparency theory resolves an old tension. Dynamic approaches are empirically powerful, but this has a serious cost: they are not predictive. The transparency theory is fully predictive and yet matches previous empirical results. That could be the end of the story: someone motivated

\footnotetext{
${ }^{3}$ LaCasse (2008) recently investigated a way to constrain the space of possible dynamic meanings for connectives. Rothschild (2008) investigated a different route. He offers a way to derive the dynamic semantics of a given expression (e.g., a conditional) from the combination of all its possible reformulations.

${ }^{4}$ Some minimal information about the order of the various pieces is also needed in the incremental version of the algorithm.

${ }^{5}$ I believe it is only for "pedagogical" reasons that this is not the route that Schlenker explores: he tries to make as easy as possible the comparison between the transparency theory and dynamic semantics.
} 
what dynamic approaches got from stipulations. Paradoxically, the impact of the transparency theory on the field is quite the opposite so far: new competing approaches to presupposition projection emerge rapidly (Chemla, 2008b; Klinedinst and Rothschild, 2008; George, 2008; LaCasse, 2008; Rothschild, 2008 and even Schlenker, 2008b). To me, this multiplication of new theories is due to the fact that Schlenker's work also reveals that:

1. A predictive theory is possible: Schlenker offers various insights towards a predictive system, in particular the processing module mentioned in (4) is now available for virtually any predictive approach. ${ }^{6}$

2. The empirical discussion is not settled. I will focus the rest of my comments on a particular aspect of this last point: projection from the scope of quantifiers.

\section{Quantified sentences}

\subsection{The data}

Sentence (6) contains a presupposition trigger, know, in the scope of the quantifier no. What is the resulting presupposition of this type of sentences?

(6) None of these 10 students knows that he is stupid.

There are two common answers to this question in the dynamic literature. ${ }^{7}$ Heim (1983) argues that (6) has the universal presupposition given in (7a). On the other hand, Beaver $(1994,2001)$ argues that it has the much weaker existential presupposition given in (7b). See Kadmon (2001, chapter 10) for discussion.

(7) a. Each of these 10 students is stupid.

b. At least one of these 10 students is stupid.

The transparency theory goes with the first camp and predicts that presuppositions triggered from the scope of a quantifier give rise to universal presuppositions. This prediction is not dependent on the quantifier, and sentences like the following are predicted to trigger the same universal presupposition:

(8) a. Less than 3 of these 10 students know that they are stupid.

b. More than 3 of these 10 students know that they are stupid.

c. Exactly 3 of these 10 students know that they are stupid.

d. Quantifier of these 10 students know that Bound pronoun is stupid.

\footnotetext{
${ }^{6}$ Interestingly, this principle is not a priori restricted to presupposition projection theories, but may also concern algorithm to compute other kinds of implicatures (e.g., scalar implicatures where this principle could add a lot to the debate between so-called globalists and localists).

${ }^{7}$ I ignore here DRT approaches. These could yield more flexible results for quantifiers which may introduce discourse referents, but probably not for others, e.g., less than 3.
} 
These predictions are problematic. From an utterance of (6), it is natural to infer that (7a) is true, but the strength of this inference decreases greatly for utterances of any of the sentences in (8). This claim is confirmed by experimental investigations described in Chemla (2008a). These data are mentioned in appendix B of Schlenker (2008a) but no solution is offered.

From the perspective of dynamic semantics, the presuppositions of quantified sentences are driven by the lexical entry of the quantifiers. In principle, it is technically possible to encode different presuppositional behaviors for different quantifiers, but this would be difficult to motivate on independent grounds and it is more parsimonious to postulate uniform presuppositional properties for all quantifiers. In a predictive framework, the difference in bivalent meanings between quantifiers could naturally come into play and explain the contrasts we observe.

So, in my view, the predictions of the transparency theory are too conservative: there is no reason to stick to a uniform treatment of quantifiers in a predictive framework. In fact, this challenge raised by quantified sentences motivated at least in part new systems of presupposition projection: Chemla (2008b) and George (2008). Alternatively, one may argue that the contrasts we see between quantifiers do not participate to the projection problem of presuppositions per se. I briefly discuss this possibility in the following section.

\subsection{Various options}

\section{Universal predictions + weakening mechanisms (difficulty?)}

Let us imagine that the universal camp is right: no matter what the quantifier is, presuppositions project universally from the scope of a quantifier. The fact that the universal inference is often rejected for certain quantifiers might be due to differences in the computations involved. For instance, it is known that downward monotonic environments lead to more difficult inferences (e.g., Geurts, 2003). Thus, one could imagine that when a presupposition trigger appears in a downward monotonic environment, a lazy hearer/speaker does not go through the whole computation process needed for proper presupposition projection and therefore does not arrive at the universal presupposition. However, the experimental data show no difference between the acceptance rate of universal presuppositions triggered from the scope of more than 3 and less than 3. If anything, Schlenker himself defends that the universal presupposition is more robust with less than 3 , i.e. in the a priori harder downward monotonic environment. (I agree with these introspective judgments although this is typically a case of rather subtle and controversial contrast where an experimental confirmation with naive speakers would be needed).

Hence, if the transparency theory is right, the lower acceptance rates of the universal inferences would have to be attributed to some difficulty in the application of the algorithm for some quantifiers but not others and we should be able to pin down the origin of this difficulty. As discussed above, it is very unlikely that it corresponds to the relative difficulty of monotonicity inferences.

Let me mention a solution which would be more specific to the transparency theory. It could be that some quantifiers require inspections of more "potential second conjuncts" (the $\beta$ mentioned in (2)) to get to the full universal prediction. In other words, it is possible that a lazy speaker does not go all the way through the examination of all the potential expressions and therefore fails to reach the universal presupposition. I leave this challenge as an open issue for the transparency theory: 
what kind of difficulty (or weakening mechanism in general) makes the presupposition weaker than expected in some quantified sentences but not others? I believe that a proper answer to this question would involve building a bridge with our understanding of general reasoning skills and thus requires proper experimental investigations.

\section{Alternatives}

There are two main empirical alternatives. First, it could be that the presupposition is the same for every quantified sentences, except that it is not universal but simply existential, as advocated by Beaver $(1994,2001)$ for instance. In this type of theories, the universal inferences could be due to pragmatic enrichments of the existential presuppositions and the application of this enrichment may depend on the overall meaning of the original quantified sentence. Such a pragmatic or probabilistic strengthening mechanism remains to be stated explicitly, just as the weakening mechanism alluded to in the previous section.

Finally, we may try to account for the differences between the various quantifiers within a predictive theory of presupposition projection. Chemla (2008b) and George (2008) offer such attempts. The challenge for this last type of approaches is to discover what the exact presuppositions of these sentences are - they might be intermediate between the existential and the universal options given in (7) -, how they vary with the bivalent meaning driven by the quantifier and yet explain why they sometimes support universal inferences.

The situation is both empirically and theoretically intricate. To determine whether the last word belongs to the theory of presupposition projection per se and which of the current approaches is on the right track, we may need to collect new kinds of data. This might require to work beyond the limits of standard linguistic methodology to collect and analyze, e.g., computation times which would inform us about the relative complexity of the relevant processes.

\section{New demands on our theory of presupposition projection}

The transparency theory sets the stage for a new departure in the study of presupposition projection (this includes second inspections of old-fashioned theories, e.g., the revival of Gazdar's system by Klinedinst and Rothschild, 2008). The system proposed in Schlenker (2008a) shows that we can hope for a predictive theory of presupposition projection; it even offers various modules which can be used to match the good old results. Consequently, the expectations for new theories rises in another major aspect: the empirical predictions should be refined together with our understanding of the data themselves. (I illustrated this point with a particular aspect of quantified sentences, although it could be extended to other cases, e.g., disjunctions and connectives in general are discussed at length in Schlenker, 2008a). Indeed, together with Schlenker's important theoretical progresses, psycholinguistic means now become available to investigate more subtle empirical data, and to draw bridges between linguistic knowledge and more general pragmatic and reasoning abilities. 


\section{References}

Beaver, D. (1994). When variables don't vary enough. In Harvey, M. and Santelmann, L., editors, Semantics and Linguistic Theory 4. CLC Publications, Cornell.

Beaver, D. I. (2001). Presupposition and assertion in dynamic semantics. CSLI Publications.

Chemla, E. (2006). Aren't Dummy Alternatives only Technical Shortcuts? Ms., ENS \& MIT.

Chemla, E. (2008a). Presuppositions of quantified sentences: Experimental Data. Under revision for Natural Language Semantics.

Chemla, E. (2008b). Similarity: Towards a unified account of scalar implicatures, free choice permission and presupposition projection. Under revision for Semantics and Pragmatics.

Gazdar, G. (1979). Pragmatics: Implicature, Presupposition and Logical Form. Academic Press, New York.

George, B. R. (2008). Presupposition repairs: a static, trivalent approach to predict projection. Master's thesis, UCLA.

Geurts, B. (2003). Reasoning with quantifiers. Cognition, 86(3):223-251.

Heim, I. (1983). On the projection problem for presuppositions. Proceedings of WCCFL, 2:114125.

Kadmon, N. (2001). Formal Pragmatics: Semantics, Pragmatics, Presupposition, and Focus. Blackwell Publishers.

Klinedinst, N. and Rothschild, D. (2008). Presuppositon projection, again. Ms. UCL and Columbia University.

LaCasse, N. (2008). Constraints on connectives and quantifiers. Ms. UCLA.

Rothschild, D. (2008). Making dynamics semantics explanatory. Ms. Columbia University.

Schlenker, P. (2007). Anti-dynamics: presupposition projection without dynamic semantics. Journal of Logic, Language and Information, 16(3):325-356.

Schlenker, P. (2008a). Be Articulate: A Pragmatic Theory of Presupposition Projection. Theoretical Linguistics.

Schlenker, P. (2008b). Local Contexts. Ms. IJN and NYU.

Soames, S. (1989). Presupposition. In Gabbay, D. and Guenther, F., editors, Handbook of Philosophical Logic, volume IV, pages 553-616. Reidel, Dordrecht. 